\title{
POLARIZATION AND MAGNETIC FIELDS IN SUPERNOVA REMNANTS
}

\author{
D.K.MILNE \\ The Australia Telescope National Facility* \\ CSIRO, Epping, NSW, 2121 \\ Australia
}

\begin{abstract}
Observations of polarization in and around Galactic supernova remnants, the deduced Faraday rotation and magnetic fields and their interpretation are reviewed. There are some indications of interaction with the Galactic magnetic field, but the conclusions are that this is an aspect which would benefit from considerably more observation and theoretical modelling.
\end{abstract}

\section{Introduction.}

It is not clear from current knowledge of the supernova event or the subsequent expansion of the remnant into the interstellar medium, just how much influence is derived from the interstellar magnetic field nor how much the supernova remnant (SNR) contributes to the Galactic field. In this brief review we outline what is known of the SNR magnetic field.

Supernova remnants are clearly synchrotron emitters and as such they contain magnetic fields. This was demonstrated by I.S. Shklovsky in 1953 to explain the optical polarization in the Crab Nebula. However the Crab Nebula is the only Galactic SNR known to have optical synchrotron emission and our knowledge of SNR magnetic fields is derived almost exclusively from their radio emission.

In its simplest form, with uniform magnetic field $\mathbf{B}$, the intensity of synchrotron emission at frequency $v$ is given by:

$\mathrm{I}_{v} \propto \mathrm{K} \mathbf{B}(\gamma+1) / 2 v^{-(\gamma-1) / 2}$, where $\mathrm{K}$ and $\gamma$ describe the relativistic electron energy distribution. $\gamma$ can be obtained from the radio spectral index $\alpha$ (i.e. $\gamma=1-2 \alpha, \alpha<0$ ), but generally $\mathrm{K}$ is not known and so B can only be inferred from the equipartition argument, magnetic energy $(B / 8 \pi)=$ kinetic energy $(3 \mathrm{kT})$. It would then appear that SNR magnetic fields range from milligauss in the Crab Nebula filaments, down to microgauss, a few times the general Galactic value, in most others. Where the magnetic field has been deduced other than by equipartition the agreement with equipartition was good (e.g. the Crab Nebula, B 0.3 mG (Kennel and Cornniti 1984) and Kepler's SNR, B 70 $\mu \mathrm{G}$ (Matsui et al. 1984)).

It was argued by van der Laan (1962) that the increased magnetic field in SNRs was derived from compression of the ambient interstellar field and cosmic ray electrons, and certainly he was able to account for the magnetic field and emissivity in the Cygnus Loop with a compression ratio of four. The magnetic field in the Cygnus Loop is tangential (i.e. it runs around the shell) whereas many other SNRs (e.g. Cas A and Tycho's) display a radial field. Whiteoak and Gardner (1968) explained this in terms of viewing angle but the increasing number of SNRs with radial fields raised statistical objections. In addition the high emissivity of the younger SNRs rules out compression of the ambient medium as the source of relativistic electrons and magnetic field. The van der Laan model is still valid for the older remnants where we do see tangential fields and lower emissivities. However a new mechanism was to be found for the radial fields present in the younger remnants.

* The Australia Telescope National Facility is operated in association with the Division of Radiophysics by CSIRO 


\section{SNR Evolution.}

Woltjer (1972) proposed a four phase dynamical model for the SNR evolution. This simple model provided a good starting point but it neglected several important factors, paramount of which were: the effect of inhomogeneities in the ISM, continued energy input from a central pulsar, non-isotropic ejection and the effect of magnetic fields both within and around the SNR. The 1970's saw the construction of several analytical and numerical models of SNR dynamics to include these effects. The model that emerged ihas the following components:

(a) as the ISM is swept up and the density of the shell increases a shock-wave will propagate outwards into the ISM from the impact surface and a reflected reverse shock will travel back into the expanding ejecta (Chevalier 1976);

(b) the model is further complicated by the cloudy nature of the ISM. McKee and Cowie (1975) considered a two phase ISM with cool dense clouds imbedded in a warm thin medium. The impinging shock will move more slowly into the dense clouds and will reach the radiative phase earlier than elswhere, the clouds will radiate in X-rays and, if cool enough, at optical wavelengths;

(c) the reverse shock will also move back through the clouds resulting in evaporation of the clouds which will increase the material behind the shock and may lead to a departure from the Sedov expansion, $R \sim t$ 2/5 ; phase II of Woltjer's model (Cowie and McKee 1977).

(d) finally there are likely to be isolated high-temperature low-density tunnels (Cox and Smith 1974) and wind-swept cavities (Braun and Strom 1986) into which the remnant can expand, changing its dynamical evolution and morphological shape.

Figure 1. A sketch to illustrate the expansion of a young SNR as discussed in the text. Attempts have been made to include the fronts, cool dense clouds in Taylor instabilities and stretching and compression of the magnetic fields. forward and reverse shock a warm medium, Rayleigh-

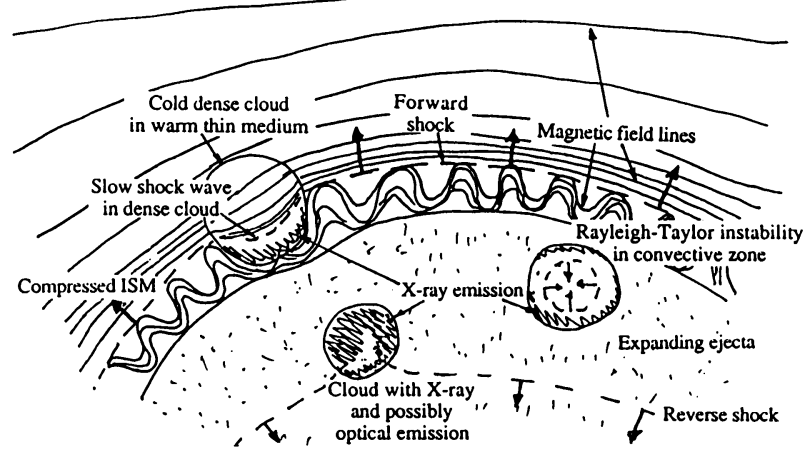

Magnetic fields are expected to be amplified by turbulence and the relativistic particles will be accelerated by the Fermi process. It is further expected that there will be radial magnetic field alignment due to Rayleigh-Taylor instabilities in the convective-like motions at the contact surface between the ejected matter and the swept-up ISM (Gull 1973). There will also be amplification of the interstellar (Galactic) magnetic field frozen into the compressed gas behind the shock front (van der Laan 1962). Magnetic fields can also modify thermal plasma conduction, i.e. conduction along the field is unimpaired but across the field is inhibited, so the magnetic geometry is important and may delay the time at which the remnant becomes radiative; this has been advanced as an explanation of the large Galactic loops like the North Polar Spur. Magnetic geometry may also be a factor in shaping highly symmetrical SNRs such as G327.6 + 14.6 (SN1006) and G296.5 + 10.0 (Roger et al. 1988).

A recent modelling of the early stages of expansion, to include magnetic fields (Dickel et al. 1989), indicates that turbulent amplification of the magnetic field at the Rayleigh-Taylor unstable surface is the most important factor in the overall radio morphology, but further, that it is necessary to include a cloudy ISM to produce radial field alignment. 
Clearly the Crab Nebula is a special case, and with the discovery of a pulsar at the centre it was proposed that this provided a continuing energy source within such SNRs and that the observed magnetic fields were wound-up pulsar generated field (Rees and Gunn 1974).

\section{Types of SNR.}

It has become fashionable to divide SNRs into three classes on the basis of their morphology and radio spectral index, they are:

(a) steep-spectrum, shell-type remnants in which the radio emission is presumed to originate from relativistic particles and magnetic fields generated either in the explosion (Shklovsky 1960) or during the early stages of the expansion (Gull 1973), or swept up from the interstellar medium (van der Laan 1962);

(b) flat-spectrum, filled-centre (plerionic or Crab Nebula-like) remnants, which require a continuing source of relativistic particles, such as a pulsar, to sustain the optical and X-ray emission (Rees and Gunn 1974);

(c) the composites, in which a compact plerionic component is found imbedded in a shell. It is possible that eventually all remnants develop a shell and that the composites are a stage in their evolution (Lozinskaya 1980).

The shells generally exhibit less polarization than plerions and have much more complex magnetic field structures. Polarization is typically $<5 \%$ but may reach $20-30$ per cent in a few shell SNRs.

\section{SNR Polarization.}

SNR emission, by its spectrum and polarization, is clearly synchrotron. In this process the linear polarization electric vector will be aligned orthogonally to the magnetic field and consequently a knowledge of the direction of the polarization allows us to determine the direction of the projected magnetic field.

The picture is complicated by Faraday rotation of the polarization. This rotation will be proportional to wavelength squared in the simplest case where the Faraday rotation is in front of the emission region and will follow a more complicated relationship if the emission and rotation are from the same region (Burn 1966). The constant of proportionality is the Rotation Measure (RM) and is given by the product of the electron density and the component of magnetic field along the line of sight, i.e. $R M=8.1 \times 10^{5} \int_{L} N e B_{L} d L$.

Synchrotron emission from electrons in a uniform magnetic field will be linearly polarized at a degree (independent of frequency) given by $\mathrm{P}=(3-3 \alpha) /(5-3 \alpha)$, where $\alpha$ is the spectral index of the radiation; for $\alpha \sim-0.5$, typical of SNRs, $P=0.7$.

However there are various effects which can reduce the observed polarization, e.g. (i) if the magnetic field is not uniform the degree of polarization will be reduced to $P / \sqrt{n}$, where $n$ is the number of polarization cells in the beam, (ii) the observed polarization may be decreased even in the presence of a uniform field if there is differential Faraday rotation (by thermal electrons) of emission from different depths; models for this mechanism have been explored by Burn (1966) and Sazonov (1973) and (iii) the polarization may be further reduced by differential Faraday rotation across the telescope beam (Milne 1980). For details of the various instrumental effects see Milne and Dickel (1975).

Linear radio polarization has been mapped using various techniques from detecting the total power modulation with feed rotation, switching between or correlating linear orthogonal signals and more recently quadrature correlation of R.H. and L.H. circular polarization as well as interferometric and 
synthesis techniques. Amongst the earliest observations of SNR radio polarization were the Mayer and Hollinger (1968) maps of Cas A and the Crab Nebula and observations of the Vela SNR (Milne 1968). In the first of these the signals were very strong and, working at only one fairly short wavelength $(1.55 \mathrm{~cm})$, Mayer and Hollinger were able to show fairly convincingly that Cas A, a young shell-remnant, has a radial magnetic field. Milne used three much longer wavelengths to estimate the Faraday rotation and show a projected field looping around the brighter parts of the old Vela SNR in a near tangential direction. This "radial in the young and tangential in the old" model has persisted and has been reinforced ever since.

To date there are polarization observations of about 80 SNRs, about half of the known SNRs in our Galaxy. Most of the older, larger remnants, have been mapped using either the NRAO 140-ft and 300-ft telescopes, the Parkes 64-m telescope or the Effelsberg 100-m telescope. There is a distinct advantage in using alt-azimuth mounted antennas, such as these latter two, since telescope deflections and ground radiation are both altitude dependent and therefore easier to correct. Observations of many of the smaller, often younger, SNRs have been made with synthesis arrays, at Cambridge, Westerbork, or the VLA. With interferometric polarization measurements it is important to obtain the short spacings since the broad structure, the radial and tangential components, could be lost (e.g. Wright and Forster 1980).

Examples of recent single-dish observations are to be found in: Haslam et al. (1980), Milne (1980), Reich and Braunsfurth (1981), Fürst et. al. (1984), Reich et. al. (1986), Roger et. al. (1986), Milne et.al. $(1986,1989)$ and the references therein. Most of the recent synthesis polarization observations have been made with the VLA, e.g. Becker and Szymkowiak (1981), Matsui et al. (1984), Velusamy (1985), Shaver et al. (1985) and Dickel et al. (1988). In Figure 2 an example of a singledish observation of a field containing two SNRs is shown, note the structure in the background polarization (Milne et al. 1989).

It seems that as the resolution increases, the polarization structure becomes more complex. We find that most SNR polarization is located in domains bounded by abrupt changes in polarization direction. In a few remnants e.g. The Vela SNR (Milne 1980) and the Crab Nebula (Swinbank 1980), there is a hint that these domains are bounded by the optical filaments. In Vela this is possibly easier to understand since it would appear that the arc-like filaments are the envelopes of bubbles in the SNR shell. Certainly in the Crab Nebula, Swinbank (1980) and Velusamy (1985) find that the depolarization occurs along the near-side filaments. An explanation implying that Rayleigh-Taylor instabilities can create filaments has been given by Reynolds (1988). In the older SNRs, these domains are often regions of uniform rotation measure and the boundaries are lines of high rotation measure gradients, which of course can further depolarize the emission by differential Faraday rotation across the telescope beam (Milne 1980).

Some attempts have been made to assess the size of the turbulent cells from the spatial correlation widths of rotation measure in Tycho's SNR (Lerche and Caswell 1979) and in the Vela SNR (Lerche and Milne 1980). Findings are that the cells are of the order of the SNR radius ( $2.6 \mathrm{pc}$ at $2.3 \mathrm{kpc}$ ) in the young Tycho SNR whilst, in contrast, in the old Vela SNR they are only about one fifth of the radius, but still approximately the same physical size $(\sim 2 \mathrm{pc})$. A promising correlation has been found between RM and the square root of the X-ray surface brightness (Matsui et al. 1984) which gave an estimate of the magnetic field strength in Kepler's SNR independent of equipartition arguments, as mentioned in section 1.

Attempts to fit the rotation and depolarization to Burn's (1966) models have generally been inconclusive owing to the small number of frequencies where data are available. There are only about 30 remnants for which we have sufficient polarization data to fit rotation measures to obtain magnetic field directions. In spite of the domain structure in many of the polarization maps, the magnetic fields are surprisingly uniform showing mostly radial fields, a few which could be described as tangential and many where the field simply sweeps across the remnant. 


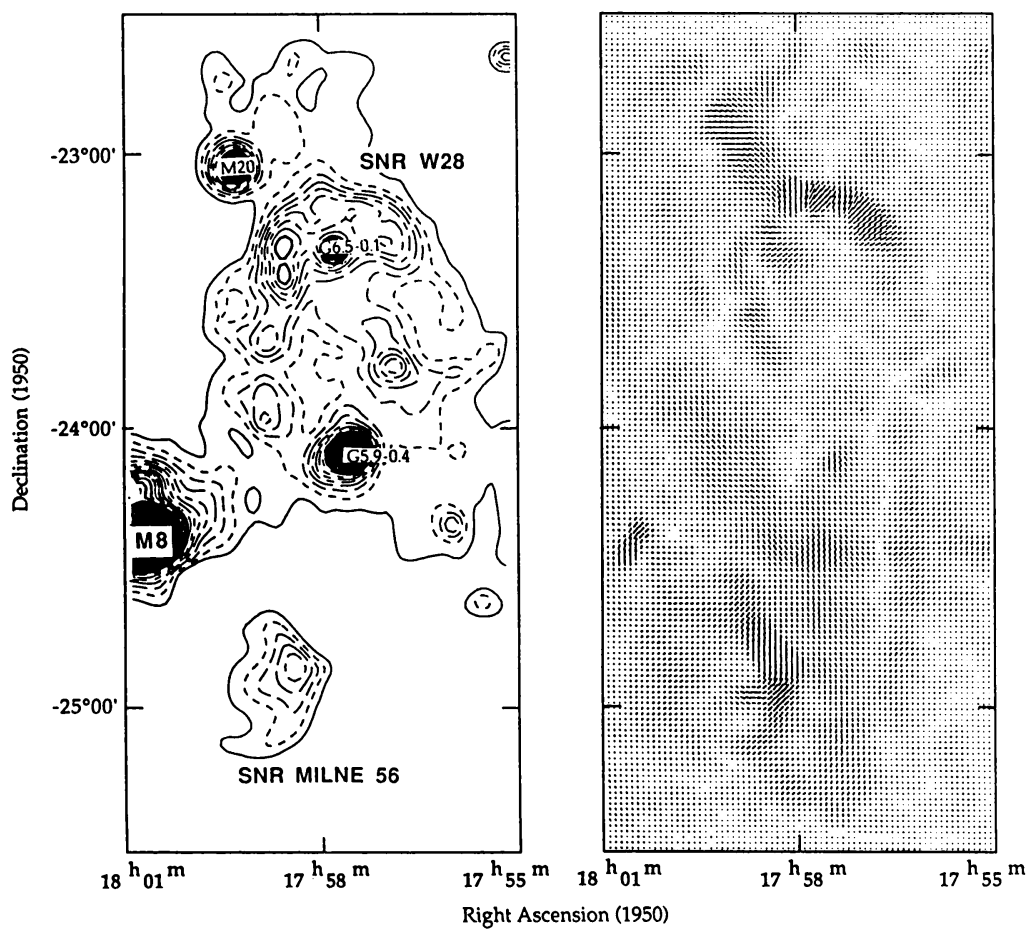

Figure 2. Preliminary maps of the $8.4 \mathrm{GHz}$ total intensity and polarization $\mathbf{E}$ vectors in a field containing two SNRs, Westerhout 28 and Milne 56 (G5.4-1.2), observed at Parkes using a quadrature correlation receiver on the $64 \mathrm{~m}$ telescope (Milne et. al. 1989). Considerable background structure is shown in the polarization map, including a faint ring to the north of W28, possibly an old very faint SNR. The four very bright sources are $\mathrm{HII}$ regions, with $\sim 0.5 \%$ instrumental polarization seen on the brightest, M8.

\section{Effects Involving the Galactic Magnetic Field.}

Certainly the SNRs are the brightest features in polarization maps of our Galaxy (e.g. Junkes et al. (1987). However, there are patches of substantial polarization where there are no known SNRs and many maps of SNR polarization do exhibit polarization seemingly extending beyond the bounds of the SNR as well as isolated polarized regions (e.g. Milne and Dickel 1975). In addition there is evidence that magnetic fields may be blown out into adjacent windswept cavities (Milne et al. 1989).

Caswell and Lerche (1979) found that there was brightening of the shell SNRs on the side nearest the Galactic plane, where the ISM was more dense. It was expected that there would also be some alignment due to the Galactic magnetic field, and indeed Roger et al. (1988) believe that there is magnetic shaping in highly symmetrical SNRs. Current revisions to "An Atlas of Supernova Remnant Magnetic Fields" (Milne 1987) to include recent Parkes 64-m observations, indicates that there are a few more remnants (12 in all) which exhibit tangential fields. Of these, perhaps eight exhibit a general field alignment to the Galactic plane, which deserves further investigation.

Finally, Sarkar (1982) suggests that localized regions of high emissivity in the non-thermal background are the shells of old SNRs in which the emissivity is enhanced by compression of the interstellar magnetic field and cosmic ray electrons. This is consistent with observations implying large amounts of hot, thin gas in the Galaxy, so that SNRs expand to large radii before becoming 
radiative. In his model, the fluctuating component of the Galactic magnetic field then arises from distortion of the regular field by the SNR shells.

\section{REFERENCES.}

Becker,R.H., Szymkowiak,A.E. (1981). Astrophys. J. 248, L23.

Braun.R., Strom,R.G. (1986). Astron. Astrophys. 164, 208.

Burn,B.J. (1966). Mon. Not. R. Astron. Soc. 133, 67.

Caswell,J.L., Lerche,I., (1979). Mon. Not. R. Astron. Soc. 187, 201.

Chevalier,R.A. (1976). Astrophys. J. 207, 872.

Cowie,L.L., McKee.C.F. (1977). Astrophys. J. 211, 135.

Cox,D.P., Smith,B.W. (1974). Astrophys J. 189, L125.

Dickel,J.R., Eilek,J.A., Jones,E.M., Reynolds,S.P. (1989). Astrophys J. Suppl. (in press)

Dickel,J.R., Sault,R., Arendt,R.G., Matsui,Y., Korista,K.T. (1988). Atsrophys. J. 330, 254.

Fürst,E., Reich,W., Streube,R. (1984). Astron. Astrophys. 133, 11.

Gull,S.F. (1973). Mon. Not. R. Astron. Soc., 161, 47.

Haslam,C.G.T., Pauls,T., Salter,C.J. (1980). Astron. Astrophys. $92,57$.

Junkes,N., Fürst,E., Reich,W.,(1987). Astron. Astrophys. Suppl. Ser. 69,451.

Kennel,C.F., Coroniti,F.V. (1984). Astrophys. J. 283, 710.

Lerche,I., Caswell,J.R. (1979). Astron. Astrophys. 77, 117.

Lerche,I., Milne,D.K. (1980). Astron. Astrophys. 81, 302.

Lozinskaya,T.A. (1980). Astron. Zh., 57, 707.

Matsui,Y., Long,S.K., Dickel,J.R., Greisen,E.R. (1984). Astrophys. J. 287, 295.

Mayer,C.H., Hollinger,J.P. (1968). Astrophys. J. 151, 53.

McKee,C.F., Cowie,L.L. (1975). Astrophys. J. 195, 715.

Milne,D.K. (1968). Aust. J. Phys. 21, 201.

Milne,D.K. (1980). Astron. Astrophys. 81, 293.

Milne,D.K. (1987). Aust. J. Phys. 40, 771.

Milne,D.K., Caswell,J.L., Kesteven,M.J., Haynes,R.F., Roger,R.S. (1989). "Supernova Shells and their Birth Events", ed. W Kundt, Berlin, Springer, Lectures Notes in Physics, 316, 98.

Milne,D.K., Caswell,J.L., Kesteven,M.J., Haynes,R.F., Roger,R.S., (1989). Proc. Astron. Soc. Aust. (in Press)

Milne,D.K., Dickel,J.R. (1975). Aust. J. Phys. 28, 209.

Milne,D.K., Haynes,R.F., Caswell,J.L., Kesteven,M.J. (1989). "Magnetic Fields in the the Supernova Remnants W28 and Milne 56" - in preparation.

Milne,D.K., Roger,R.S., Kesteven,M.J., Haynes,R.F., Wellington,K.J., Stewart,R.T. (1986) Mon. Not. R. Astron. Soc. 223, 487.

Rees,M.J., Gunn,J.E. (1974). Mon. Not. R. Astron. Soc. 167, 1.

Reich,W., Braunsfurth,E. (1981). Astron. Astrophys. 99, 17.

Reich,W., Fürst,E., Reich,P., Sofue,Y., Handa,T. (1986). Astron. Astrophys. 155, 185.

Reynolds, S., (1988). Astrophys. J. 327, 853.

Roger,R.S., Milne,D.K., Caswell,J.L., Little,A.G. (1986). Mon. Not. R. Astron. Soc. 133, 67.

Roger,R.S., Milne,D.K., Kesteven,M.J., Wellington,K.J., Haynes,R.F. (1988) Astrophys. J. 332, 940.

Sarkar,S. (1982). Mon. Not. R. Astron. Soc. 199, 97.

Sazonov,V.N. (1973). Sov. Astron. 16, 774.

Shaver,P.A., Salter,C.J., Patnaik,A.R., van Gorkom,J.H., Hunt,G.C. (1985). Nature 313, 113.

Shklovsky,I.S. (1960). Sov. Astron. 4, 243.

Swinbank,E. (1980). Mon. Not. R. Astron. Soc. 193, 451.

Van der Laan, H. (1962). Mon. Not. R. Astron. Soc., 124, 125.

Velusamy,T. (1985). Mon. Not. R. Astron. Soc. 212, 359.

Whiteoak,J.B., Gardner,F.F. (1968). Astrophys. J., 154, 807.

Woltjer,L., (1972). Ann. Rev. Astron. Astrophys. 10, 129.

Wright,M., Forster,J.R. (1980). Astrophys. J. 239, 873. 